\title{
CAMADAS DE COBERTURA METANOTRÓFICAS COMO ALTERNATIVAS PARA GERENCIAMENTO DE GASES DE EFEITO ESTUFA EM ATERROS SANITÁRIOS
}

\author{
Régia Lúcia Lopes \\ Professora do Instituto Federal de Educação Ciência e Tecnologia do RN (IFRN), Campus \\ Central, desde 1991. Engenheira Civil (UFRN). Doutoranda em Engenharia Civil \\ (Geotecnia Ambiental), Membro do Núcleo de Processamento Mineral e de Resíduos \\ (IFRN) e do Grupo de Resíduos Sólidos (UFPE). Email:regia.lopes@ifrn.edu.br. \\ José Fernando Thomé Jucá \\ Professor do Departamento de Engenharia Civil da Universidade Federal de Pernambuco \\ (UFPE). Engenheiro Civil (UFPE). Doutor pela Universidad Politécnica de Madrid. \\ Coordenador do Grupo de Resíduos Sólidos (GRS) da UFPE. Diretor do Centro de \\ Tecnologias Estratégicas do Nordeste (CETENE). Email: jucah@ufpe.br.

\section{Maria Odete Holanda Mariano} \\ Professora do Departamento de Engenharia Civil da Universidade Federal de Pernambuco, \\ Campus Avançado do Agreste (CAA). Doutora em Engenharia Civil (Geotecnia \\ Ambiental), Membro do Grupo de Resíduos Sólidos (UFPE). Email: odete@ufpe.br. \\ Felipe Jucá Maciel \\ Engenheiro Civil (UFPE). Doutor em Engenharia Civil (Geotecnia Ambiental). Membro \\ do Grupo de Resíduos Sólidos (UFPE). Email: maciel2@ hotmail.com.
}

\section{RESUMO}

Os aterros sanitários representam uma fonte potencial de geração de gases de efeito estufa (GEE) para atmosfera, causada por sistemas inadequados de coleta e tratamento do biogás e emissões descontroladas pelas camadas de cobertura. Nos últimos anos tem sido pesquisadas camadas de cobertura que favorecem a oxidação do metano $\left(\mathrm{CH}_{4}\right)$, nos casos onde a captação e exploração desse biogás não sejam economicamente viáveis. Neste sentido, as camadas de cobertura mais conhecidas são as denominadas metanotróficas, que funcionam com base em determinadas características do solo, presença de $\mathrm{CH}_{4}$ e microorganismos, de forma a oxidar esse gás ao longo do perfil do solo. Esse trabalho apresenta os resultados do estudo realizado na camada de cobertura do tipo metanotrófica implantada na Célula Experimental localizada no Aterro da Muribeca, Recife-PE. A metodologia utilizada para na análise de emissões de biogás foi baseada em ensaios de campo para determinação do fluxo de metano e gás carbônico. Além disso, foram feitas medidas de concentração desses gases a cada $10 \mathrm{~cm}$, desde a interface solo/resíduo até a superfície, e coletadas amostras de solo para avaliar a influência das características físico-químicas do material na retenção e/ou oxidação do $\mathrm{CH}_{4}$. Os resultados têm demonstrado uma emissão de $\mathrm{CH}_{4}$ variando de 0 a $151,95 \mathrm{~g} / \mathrm{m}^{2}$.dia e uma redução da concentração de $\mathrm{CH}_{4}$ baseada na relação $\mathrm{CO}_{2} / \mathrm{CH}_{4}$, variando de $34 \%$ a $100 \%$, desde a interface solo/resíduos até a superfície. Dessa forma, as camadas do tipo metanotrófica ou oxidativas têm sido mais recentemente, utilizadas como alternativas às camadas convencionais de solo compactado, para o gerenciamento de emissões de gases de efeito estufa em aterros sanitários.

PALAVRAS-CHAVE: Aterro de resíduos sólidos, camadas de cobertura, camada metanotrófica, oxidação de metano. 


\title{
METHANOTROPHIC COVER LAYERS AS ALTERNATIVES FOR MANAGEMENT OF GREENHOUSE GASES FROM LANDFILLS
}

\begin{abstract}
Landfills are considered a potential source of the greenhouse gases emissions to the atmosphere, caused by inadequate systems for collection and treatment of biogas emissions and uncontrolled emission from cover layers. In the last years have been studied cover layers that favor the oxidation of methane, in cases where the capture and exploitation of biogas are technically difficult and very costly. The called methanotrophic cover layers used the soil characteristics, presence of methane and microorganisms in order to oxidize the gas throughout the soil profile, and they are been considering alternatives to minimize the emission of methane from landfills. This paper presents the results of a study conducted in methanotrophic cover layers located in Experimental Cell in the Muribeca's Landfill, Recife Brazil. The methodology for the analysis of biogas emissions was based on field tests for determining the flow of methane and carbon dioxide. In addition, measurements of concentration of biogas were made every $10 \mathrm{~cm}$ from the soil/waste to the surface, and collected soil samples to assess the influence of some physical and chemical characteristics of the material in the retention and/or oxidation of methane. The results have shown an emission of methane ranging from 0 to $151.95 \mathrm{~g} / \mathrm{m}^{2}$.dia and a reduction of methane concentration on the relationship between $\mathrm{CO}_{2}$ and $\mathrm{CH}_{4}$, ranging from $34 \%$ to $100 \%$, from the soil/waste until the surface. Thus, the methanotrophic layers have more recently been used as alternatives to conventional layers of compacted soil, for managing emissions of greenhouse gases in landfills.
\end{abstract}

KEY WORDS: Landfills solid waste, cover layers, methanotrophic cover, methane oxidation. 


\section{CAMADAS DE COBERTURA METANOTRÓFICAS COMO ALTERNATIVAS PARA GERENCIAMENTO DE GASES DE EFEITO ESTUFA EM ATERROS SANITÁRIOS}

\section{INTRODUÇÃO}

A disposição de resíduos sólidos urbanos (RSU) em aterros sanitários vem crescendo no Brasil nas últimas décadas. De acordo com a Pesquisa Nacional de Saneamento Básico (PNSB) divulgada recentemente, a utilização de lixões e vazadouros a céu aberto caiu de $72,3 \%$ para 50,2\% dos municípios brasileiros e o lançamento em aterros sanitários cresce de $17,3 \%$ para $27,7 \%$ entre os anos 2000 a 2008 (IBGE, 2008).

Os RSU quando lançados nos aterros sanitários, geram, através do processo de biodegradação anaeróbia, subprodutos em forma de líquidos e gases que precisam ser tratados e/ou aproveitados, de forma a equacionar os problemas causados pelo lançamento no solo ou em corpos hídricos receptores e na atmosfera, gerando degradação ambiental.

Os gases gerados pela biodegradação de resíduos em aterros sanitários são compostos basicamente por metano $\left(\mathrm{CH}_{4}\right)$ e gás carbônico $\left(\mathrm{CO}_{2}\right)$, sendo esses os principais gases responsáveis pelo aquecimento global, denominados gases de efeito estufa (GEE). As emissões globais de biogás de aterros são estimadas pela Agencia de Proteção Ambiental Americana (USEPA) entre 500 a $800 \mathrm{MtCO}_{2} \mathrm{eq} /$ ano e segundo o Painel Intergovernamental de Mudanças Climáticas (IPCC) em um horizonte de mais de 100 anos, o poder de aquecimento do $\mathrm{CH}_{4}$ é 21 vezes maior do que o CO2 (IPCC, 2007).

No Brasil, os resíduos sólidos urbanos representam cerca de $12 \%$ das fontes emissoras de de gás $\mathrm{CH}_{4}$, sendo a disposição final responsável por $84 \%$ desse valor, de acordo com o Inventário Nacional de Gases de Efeito Estufa (Van ELK, 2007 p.11). Os gases oriundos do processo de degradação dos resíduos em aterros no Brasil, geralmente coletados e tratados com a queima simples. $\mathrm{O}$ reaproveitamento e utilização de $\mathrm{CH}_{4}$ como fonte de energia vem sendo estimulados, tendo em vista a necessidade de se reduzir os efeitos do lançamento na atmosfera pelo fato desses gases serem causadores do efeito estufa.

De acordo com o estado de arte da tecnologia de aterros sanitários, de 40 a $60 \%$ do biogás gerado podem ser drenados pelos sistemas de coleta convencionais (drenos verticais). As maiores taxas são alcançadas quando se instalam sistemas de extração forçada e uma cobertura que impeça a fuga do biogás (HUMER et al., 2008). No entanto, esses sistemas são implantados geralmente quando se desejam recuperar o biogás para geração de energia. Os projetos de recuperação de biogás no Brasil se limitam, até então, a empreendimentos de grande porte, sendo necessário encontrar soluções para minimizar as emissões de gases de efeito estufa em aterros de pequeno e médio porte e projetos de recuperação de lixões e aterros controlados.

Esse trabalho apresenta os resultados de um estudo realizado em uma cobertura do tipo metanotrófica implantada em uma Célula Experimental localizada no Aterro da Muribeca, Recife-PE. As emissões foram medidas em campo no período de setembro de 2009 a fevereiro de 2010, em conjunto com medições de concentração de biogás, umidade e temperatura no perfil do solo, e determinação de $\mathrm{pH}$ e sólidos voláteis em ensaios de laboratório, com vistas a avaliar a influência das propriedades da mistura solo/composto nos processos de oxidação. Os resultados têm demonstrado uma emissão de $\mathrm{CH}_{4}$ variando 
de 0 a $151,95 \mathrm{~g} / \mathrm{m}^{2}$.dia e uma eficiência média de retenção/oxidação de $\mathrm{CH}_{4}$ variando de $34 \%$ a $100 \%$ desde a interface solo/resíduos até a superfície. Dessa forma as camadas do tipo metanotrófica se configuram como uma alternativa às camadas convencionais de solo compactado, para o gerenciamento de emissões de gases de efeito estufa em aterros sanitários.

\section{CAMADAS DE COBERTURA DE ATERROS DE RESÍDUOS SÓLIDOS}

No Brasil, a maioria dos aterros possui cobertura com camada homogênea de solo compactado, em grande parte, usando solos argilosos. As camadas de cobertura geralmente são denominadas de acordo com os materiais que as constituem em (i) homogênea ou convencional: construídas com solo, normalmente de argila, com espessuras variáveis; (ii) camadas intercaladas de solo natural argiloso e geossintéticos: usadas quando há necessidade de se impermeabilizar o topo das células, seja para melhorar a captação de gases ou por exigências ambientais; (iii) camadas alternativas: compostas por solos, ou misturas de solos com outros materiais que não são geossintéticos, com a função de melhorar o desempenho com relação a emissão de gases ou infiltração de água, e minimização de custos.

As normas brasileiras NBR 8.419/92 referentes a aterros de resíduos sólidos não perigosos, onde se insere os RSU, referem-se apenas a permeabilidade a água para camadas de proteção, não se mencionando valores para o coeficiente de permeabilidade do solo, aos gases. Essa norma também não faz menção à exigência de tipo de solo, características geotécnicas, espessura e manutenção ao longo do tempo.

De acordo com Koerner e Daniel (1997), em regiões de clima árido e semi-árido, as coberturas finais devem possuir configurações e especificações de solos diferentes das coberturas convencionais construídas em aterros de regiões de clima úmido. Isso se deve ao menor desempenho verificado nos sistemas de cobertura em solo natural (argila) em climas áridos, devido a alta evaporação, que provoca ressecamento do solo e a formação de trincas, favorecendo maiores emissões de biogás.

\subsection{Camadas metanotróficas}

As camadas de coberturas denominadas metanotróficas ou oxidativas incorporam o conceito de camadas bioreativas ou biocoberturas e estão sendo utilizadas como alternativa de gerenciamento para as emissões de gases produzidos em aterros sanitários. Segundo o IPCC (2007) elas representam uma alternativa economicamente atraente para o tratamento de emissões fugitivas de $\mathrm{CH}_{4}$. Essas camadas são projetadas com a finalidade de reduzir as emissões de $\mathrm{CH}_{4}$, utilizando-se materiais diferenciados tipo composto (produtos resultantes da compostagem) e produtos similares proveniente de tratamento mecânico biológico de resíduos (MBT), com características físico-químicas e microbiológicas específicas, que apresentam condições favoráveis para o processo de oxidação de $\mathrm{CH}_{4}$, através da ação de micro-organismos, não existindo a necessidade de grandes gastos técnicos in situ (BORJESSON e SVENSON, 1997; BEGER et al., 2005; SCHEUTZ et al., 2003; ROSE et al., 2007).

$\mathrm{O}$ processo de oxidação de metano consiste na conversão de $\mathrm{CH}_{4}$ para água, dióxido de carbono e biomassa através da atividade microbiana e segue a fórmula estequiométrica: 
Através de uma seleção controlada dos substratos desejados, do controle dos parâmetros ambientais para os micro-organismos metanotróficos e da instalação de um sistema de drenagem auxiliar abaixo da camada de cobertura, para distribuir melhor o fluxo de biogás, se pode alcançar uma taxa ótima de oxidação de $\mathrm{CH}_{4}$.

Huber-Humer et al. (2008) propõem uma configuração adequada para que haja atividade de oxidação, como mostrado na figura 1 .

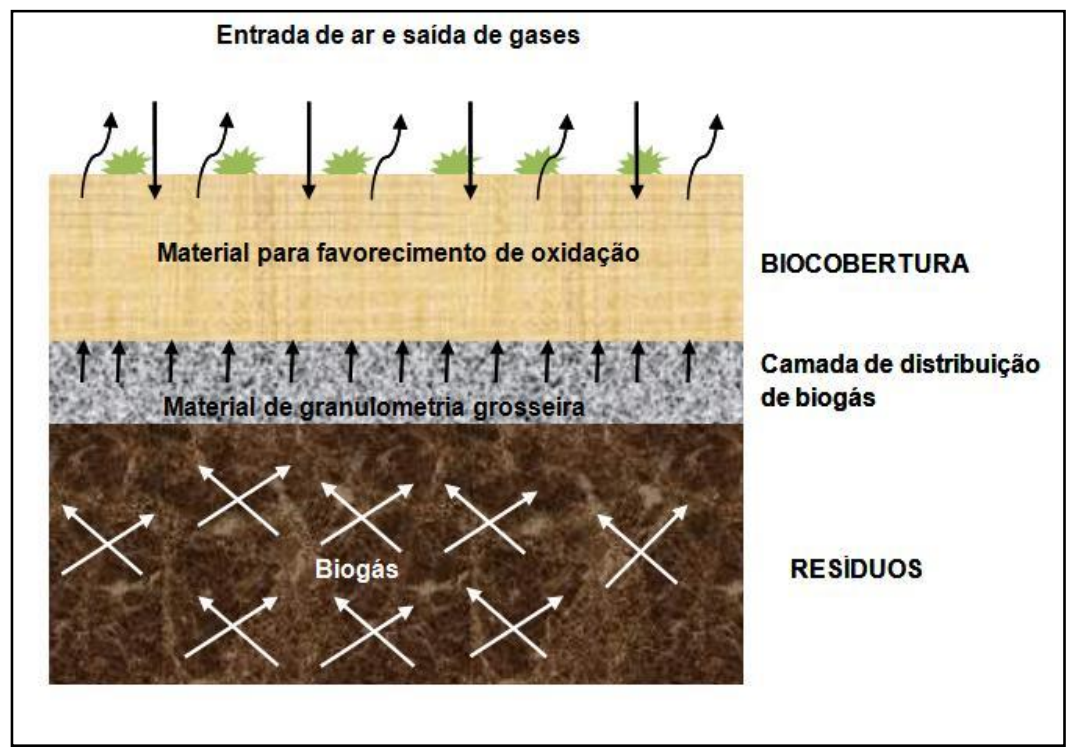

Figura 1 - Esquema conceitual de biocobertura

(Adaptado de HUBER-HUMER, 2008)

A utilização de biocoberturas implica na necessidade de avaliação de sua eficiência tanto durante a fase de planejamento quanto em toda a operação do aterro, pois a capacidade de oxidação é controlada por várias propriedades do solo, sendo os principais fatores que afetam diretamente o potencial de oxidação do $\mathrm{CH}_{4}$ : (i) espessura da camada; (ii) propriedades físico-químicas da mistura $(\mathrm{pH}$, umidade e teor de matéria orgânica); (iii) temperatura da camada de solo (IPCC, 2007). Outros fatores que também influenciam nas taxas de oxidação são concentração de $\mathrm{CH}_{4}$ sob a camada de cobertura, variáveis climáticas como temperatura ambiente, pressão atmosférica e precipitação.

Estudos de laboratório já demonstraram taxas de oxidação solos de cobertura de aterros variando de 150 a $250 \mathrm{gCH}_{4} / \mathrm{m}^{2} /$ dia, o que corresponde de $10 \pm 20 \%$ do $\mathrm{CH}_{4}$ passando pela cobertura, embora que sob condições de laboratório tem se verificado até $90 \%$ de oxidação do $\mathrm{CH}_{4}$ (BOGNER et al., 1997; WILSHUSEN et al., 2004; URMANN et al., 2007; PERDIKEA et al., 2007). Procedimentos para a quantificação in situ de $\mathrm{CH}_{4}$ e determinação exata da eficiência da oxidação no campo ainda estão em fase de desenvolvimento e estão longe de ser comercialmente disponíveis (HUBER-HUMER et al., 2009), pois normalmente, não se podem controlar os parâmetros meteorológicos em 
campo, além de que utilizam técnicas sofisticadas e onerosas, não disponíveis em países em desenvolvimento.

\section{MATERIAIS E MÉTODOS}

\section{1 Área de Estudo}

O estudo de camadas de cobertura foi realizado na célula experimental localizada na área do Aterro Controlado da Muribeca, que se situa na Região Metropolitana do Recife (RMR), no município de Jaboatão dos Guararapes-PE. A Célula Experimental implantada em 2007 faz parte de um projeto de pesquisa que tem por objetivo geral os estudos de geração de energia elétrica através do biogás de produzido pela biodegradação dos resíduos sólidos urbanos, sendo incorporadas a essa unidade diversas pesquisas na área de resíduos sólidos. A célula foi preenchida com $27.000 \mathrm{~m}^{3}$ de resíduos sólidos urbanos e para fins de pesquisa teve a cobertura fina superior composta de 3 tipos diferentes de cobertura .

\subsection{Descrição da Cobertura Experimental}

A camada metanotrófica em estudo é constituída por $30 \mathrm{~cm}$ de solo compactado sobreposto por uma camada de mistura de solo e composto orgânico na proporção de $50 \%$ em volume, correspondendo a $75 \%$ em peso de solo e $25 \%$ em peso de composto, ocupando uma área de 590,2 $\mathrm{m}^{2}$. A figura 2 mostra um perfil dessa camada com a instrumentação utilizada para medição de umidade, temperatura e concentração de biogás em profundidade.

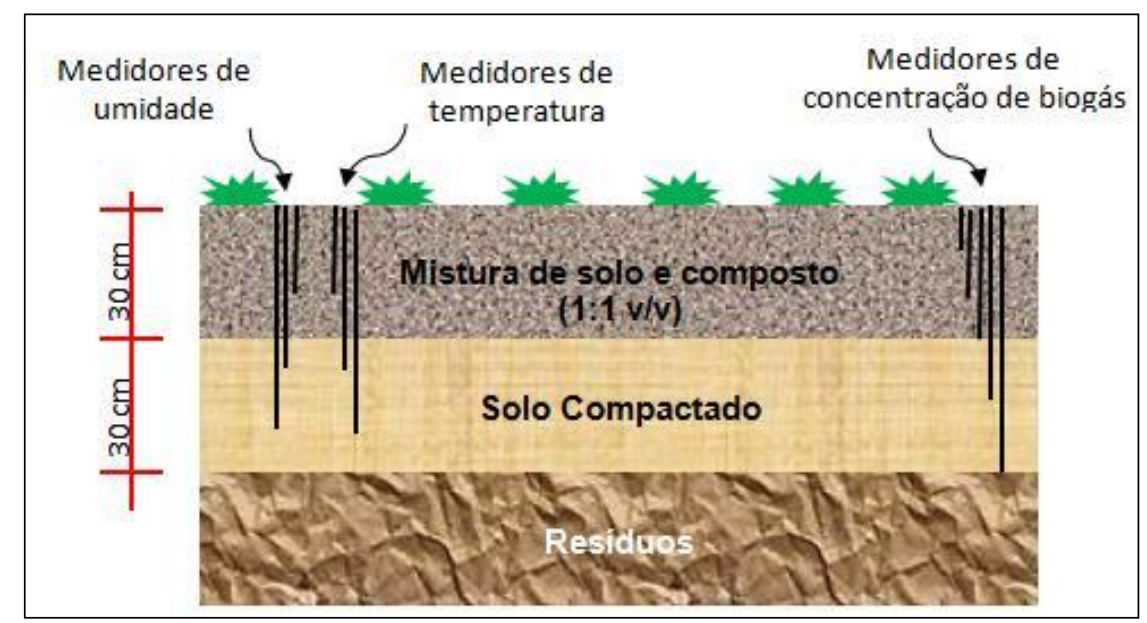

Figura 2 - Perfil da camada metanotrófica experimental

O solo e a mistura solo/composto foram caracterizados em laboratório seguindo as normas da ABNT para solos (NBR 6508/84, NBR 7181/84, NBR 6459/84, NBR 7180/84, NBR 6457/86, NBR 7182/86 e NBR 6502/95). As determinações em de pH e sólidos voláteis seguiu a metodologia indicada pela EMBRAPA (1999).

\subsection{Determinação de fluxo de biogás}


As determinações dos fluxos dos gases $\mathrm{CH}_{4}$ e $\mathrm{CO}_{2}$ através da camada metanotrófica da cobertura da célula experimental foram realizadas utilizando-se a metodologia do ensaio com placa de fluxo estática descrita por Maciel (2003) em 07 ensaios de placa de fluxo estática no período de setembro de 2009 a fevereiro de 2010.

Essa metodologia consiste na cravação cuidadosa de uma placa metálica quadrada de volume conhecido, no solo da cobertura, e na medição da concentração acumulada dos gases, temperatura e pressão dos gases (interna e externa) ao longo de um intervalo de tempo de 30 a 60 minutos. A figura 03 apresenta um desenho esquemático da placa de fluxo utilizada.

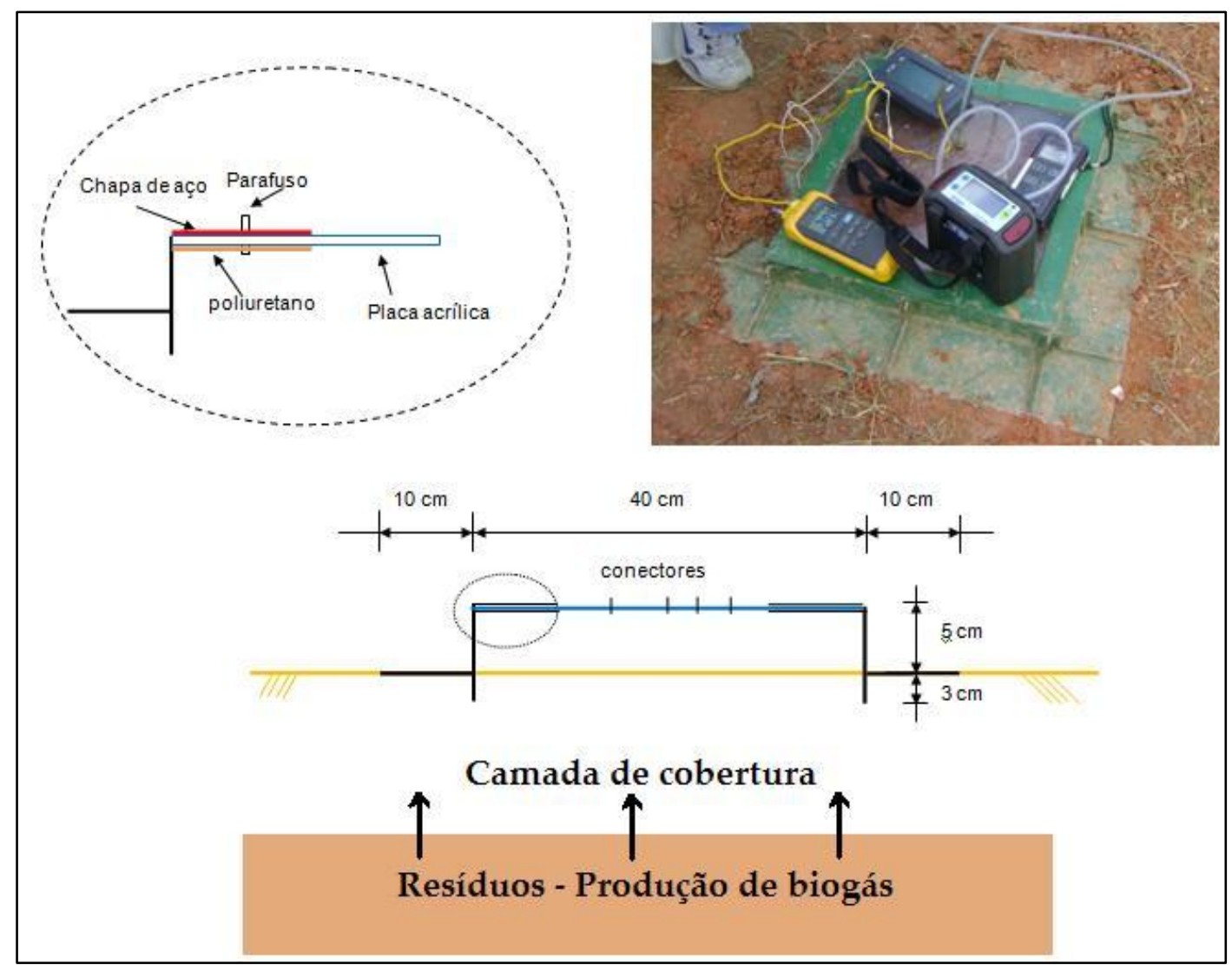

Figura 02: Corte esquemático e ensaio de placa de fluxo utilizada no estudo (Adaptado de MACIEL, 2009)

A determinação do fluxo de gás é realizada por meio da avaliação da massa (ou volume) de $\mathrm{CH}_{4}$ e $\mathrm{CO}_{2}$ aprisionada no interior da câmara, obtido a partir da inclinação inicial da curva massa $\mathrm{x}$ tempo.

\subsection{Medição de umidade temperatura e concentração no perfil da cobertura}

A umidade volumétrica era medida a partir de sensores do tipo EC5-DECAGON DEVICES instalados em 3 profundidades $(20 \mathrm{~cm}, 40 \mathrm{~cm}$ e $55 \mathrm{~cm})$ e a temperatura a partir de conector e fio termopar tipo $\mathrm{k}$, também nas 3 profundidades. A medição de 
concentração de biogás foi feita a partir de dispositivos de medição de pressão e concentração (DMPC), adaptados de Maciel (2003), instalados em 5 profundidades $(10 \mathrm{~cm}$, $20 \mathrm{~cm}, 30 \mathrm{~cm}, 40 \mathrm{~cm}$ e $60 \mathrm{~cm}$ ). As medidas de umidade em profundidade permitiram avaliar o grau de saturação médio da camada. A figura 4 mostra a instrumentação instalada e os respectivos equipamentos de medição.

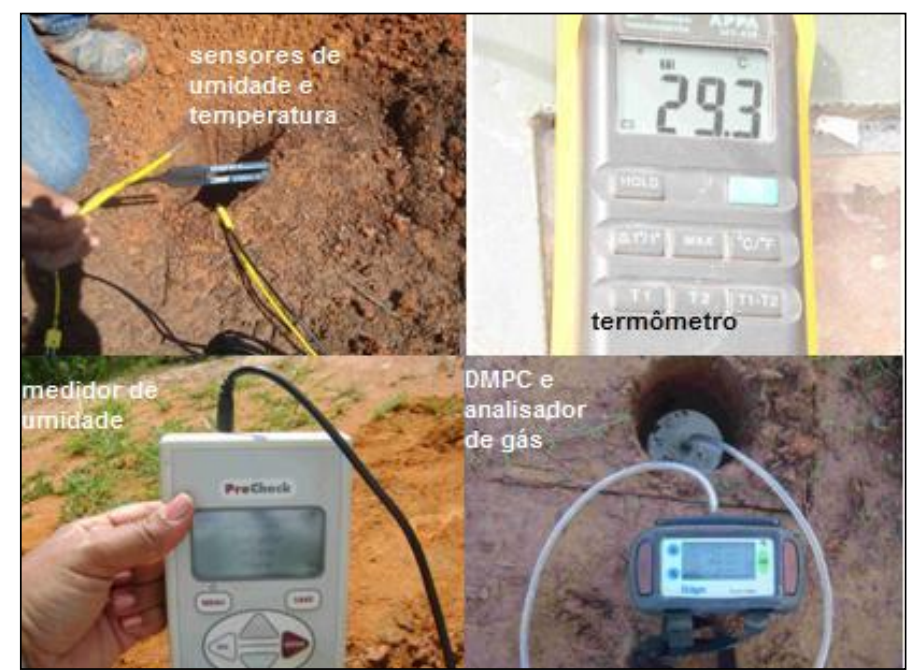

Figura 4 - Instrumentação e equipamentos utilizados

A investigação do potencial de oxidação de $\mathrm{CH}_{4}$ conjuntamente com a retenção na camada de cobertura metanotrófica foi feita com base nos perfis de concentração de $\mathrm{CH}_{4}$ e na variação da relação de concentração entre o $\mathrm{CO}_{2}$ e $\mathrm{CH}_{4}$ desde o contato solo/resíduo até a superfície do aterro (10 cm superficiais). O percentual de variação de $\mathrm{CH}_{4}$ retido e/ou oxidado ao longo do perfil é dado por:

$\operatorname{EFox}(\%)=\frac{\left(\% \mathrm{CH} 4_{\text {base }}\right)-\left(\% \mathrm{CH} 4_{\text {superficie }}\right)}{\% \mathrm{CH} 4_{\text {base }}} \times 100$ Equação (2)

Onde:

EFox = Eficiência de retenção/oxidação de $\mathrm{CH}_{4}(\%)$

$\% \mathrm{CH}_{4 \text { base }}=$ Concentração de $\mathrm{CH}_{4}$ na interface solo/resíduo (\%)

$\% \mathrm{CH}_{4 \text { superfície }}=$ Concentração de $\mathrm{CH}_{4}$ a $10 \mathrm{~cm}$ de profundidade $(\%)$

\section{RESULTADOS E DISCUSSÕES}

\subsection{Características da camada metanotrófica}

O solo utilizado na camada de cobertura foi proveniente de uma jazida localizada no próprio aterro da Muribeca e sua caracterização foi descrita em Maciel (2009). São solos com massa específica real dos grãos de $2,696 \mathrm{~g} / \mathrm{cm}^{3}$, de acordo com o Sistema Unificado de Classificação dos Solos (SUCS), são classificadas como solos areno-siltosos (SM) ou 
siltes de baixa plasticidade (ML). A média da massa específica seca máxima foi de 1,696 $\mathrm{g} / \mathrm{cm}^{3}$, compactada na energia Proctor Normal, a umidade ótima de $16,5 \%$ e a permeabilidade à água medida em laboratório com permeâmetro de carga constante foi de $2,0 \times 10^{-6} \mathrm{~cm} / \mathrm{s}$.

O composto utilizado na mistura da camada metanotrófica foi proveniente da unidade de compostagem de podas de árvores da Muribeca. O composto orgânico puro é constituído basicamente por partículas de grãos similares ao diâmetro das areias. A mistura de solo e composto na proporção de 1:1, em volume, apresentou massa específica real dos grãos $2,49 \mathrm{~g} / \mathrm{cm}^{3}$. A faixa granulométrica da mistura de solo e composto se assemelha ao diâmetro de solos areno-siltosos (SM), porém com massa específica seca máxima de 1,24 $\mathrm{g} / \mathrm{cm}^{3}$, compactada na energia Proctor Normal e umidade ótima de $33,1 \%$ devido a adição de composto que altera as características geotécnicas do material, proporcionando maior porosidade e melhor agregação de partículas em função da presença de matéria orgânica.

\subsection{Fluxo de metano}

A tabela 01 apresenta os resultados de fluxo de $\mathrm{CH}_{4}$ e gás carbônico no período analisado, na camada metanotrófica.

Tabela 01- fluxo de biogás na camada metanotrófica (setembro/09 a fevereiro/10)

\begin{tabular}{|c|c|c|c|c|c|c|c|c|c|}
\hline \multirow{2}{*}{ Ensaio } & \multicolumn{2}{|c|}{ Fluxo (g/m².dia) } & \multirow{2}{*}{$\begin{array}{c}S \\
(\%)\end{array}$} & \multicolumn{3}{|c|}{ Contato solo/resíduo } & \multirow{2}{*}{$\begin{array}{c}\Delta \mathbf{P} \\
(\mathbf{P a})\end{array}$} & \multirow{2}{*}{$\begin{array}{l}\text { Patm } \\
\text { (hPa) }\end{array}$} & \multirow{2}{*}{$\begin{array}{l}\text { GC } \\
(\%)\end{array}$} \\
\hline & $\mathrm{CH}_{4}$ & $\mathrm{CO}_{2}$ & & $\begin{array}{c}\mathrm{CH}_{4} \\
(\%)\end{array}$ & $\begin{array}{l}\mathrm{CO}_{2} \\
(\%)\end{array}$ & $\begin{array}{c}\text { O2 } \\
(\%)\end{array}$ & & & \\
\hline EP70 & 0,00 & 0,00 & 78,0 & 53,0 & 33,0 & 0,8 & nd & 1015,1 & $75 \%$ \\
\hline EP73 & 0,00 & 58,07 & 67,4 & 52,0 & 37,0 & 1,0 & 0,0 & 1014,7 & $70 \%$ \\
\hline EP89 & 35,20 & 130,48 & 57,1 & 52,0 & 39,0 & 0,8 & 6,0 & 1012,0 & $69 \%$ \\
\hline EP91 & 35,84 & 297,62 & 58,2 & 53,0 & 40,0 & 0,3 & 3,0 & 1012,3 & $75 \%$ \\
\hline EP101 & 140,27 & 405,03 & 62,5 & 57,0 & 39,0 & 0,5 & 51,0 & 1010,4 & $70 \%$ \\
\hline EP113 & 0,00 & 100,81 & 62,3 & 8,8 & 23,5 & 0,7 & 0,0 & 1013,3 & $81 \%$ \\
\hline EP117 & 151,95 & 644,74 & 56,8 & 54,0 & 33,0 & 0,5 & 48,0 & 1010,6 & $70 \%$ \\
\hline
\end{tabular}

$\mathrm{S}=$ grau de saturação no perfil da camada; $\mathrm{GC}=$ grau de compactação

Como pode ser observado nesse período, o fluxo de $\mathrm{CH}_{4}$ na camada metanotrófica medido variou de 0,00 a $151,95 \mathrm{~g} / \mathrm{m}^{2}$.dia e de gás carbônico variou de 0 a $644,74 \mathrm{~g} / \mathrm{m}^{2}$.dia.

Os ensaios EP70, EP73 e EP113 foram realizados em locais da camada onde havia intensa vegetação, apresentando fluxos nulos de $\mathrm{CH}_{4}$ e de até $100,81 \mathrm{~g} / \mathrm{m}^{2}$.dia de $\mathrm{CO}_{2}$. As concentrações de biogás no contato solo/resíduo, no EP70 e EP73 são típicas de biogás de aterros de RSU, com concentração de $\mathrm{CH}_{4}$ da ordem de 53\%. A condição de maior grau de saturação nesses dois ensaios e baixo grau de compactação favoreceu um ambiente propício para retenção de $\mathrm{CH}_{4}$ através de reações de oxidação, tendo em vista a maior possibilidade de entrada de oxigênio para as reações de oxidação. No ensaio EP113, a baixa concentração e gradiente de pressão nulo, aliada a um maior grau de compactação proporcionou a retenção total do $\mathrm{CH}_{4}$ no perfil. 
Os ensaios EP89 e EP91 apresentaram fluxos de $\mathrm{CH}_{4}$ de mesma magnitude de 35,20 $\mathrm{g} / \mathrm{m}^{2}$.dia e $35,84 \mathrm{~g} / \mathrm{m}^{2}$.dia e baixo gradiente de pressão durante os ensaios (6 e $3 \mathrm{~Pa}$ ). $\mathrm{O}$ grau de saturação médio no perfil de $57,1 \%$ e $58,2 \%$ respectivamente, com material (solo e composto) mais poroso na superfície (observação in loco), pode justificar assim a presença de fluxo nesses dois ensaios, embora de baixa magnitude, o que deve ter condicionado reações de oxidação no perfil.

Os ensaios EP101 e EP117 apresentaram fluxos de $\mathrm{CH}_{4}$ de $140,27 \mathrm{~g} / \mathrm{m}^{2}$.dia e 151,95 $\mathrm{g} / \mathrm{m}^{2}$.dia. Esses maiores fluxos apresentados foram condicionados por um maior gradiente de pressão de 51 e $48 \mathrm{~Pa}$ respectivamente, menor atmosférica observada nos dia dos ensaios, e grau de saturação em perfil de $62,5 \%$ e 56,8\% respectivamente. Embora o grau de saturação do ensaio EP101 seja superior aos ensaios com fluxos de menor magnitude (EP89 e EP91) o que pode ter acontecido é que para grau de saturação inferior a $65 \%$ em qualquer tipo de solo, a permeabilidade ao ar permanece constante com seu valor máximo, sendo influenciada por outros fatores do solo tais como tipo de solo e grau de compactação. Nesse caso provavelmente o maior gradiente de pressão e um menor grau de compactação no ensaio EP101 podem justificar um fluxo de maior magnitude.

Maciel (2003) realizando estudos de fluxos na camada de cobertura convencional do aterro controlado da Muribeca observou fluxos variando entre 103,7 g/m².dia a 362,9 g/m².dia em 6 ensaios realizados entre março e maio de 2003, com condições climatológicas similares a essa pesquisa e umidade superficial de campo variando de $16,4 \%$ a $23,7 \%$ e solo da mesma jazida. Mariano (2008) realizando estudos no aterro controlado de Aguazinha-Olinda-PE, localizado na região Metropolitana de Recife, determinou em ensaios de fluxo, variação desde fluxo nulo até $401 \mathrm{~g} / \mathrm{m}^{2}$.dia, comprovando que a camada metanotrófica apresentou menor variação de emissões de $\mathrm{CH}_{4}$ do que as camadas convencionais mencionadas nessas duas pesquisas.

\subsection{Variações de umidade, temperatura e concentração de biogás em profundidade}

No período de setembro de 2009 a fevereiro de 2010 foram realizadas 15 medições de concentração de biogás em profundidade, conjuntamente com medições de umidade e temperatura. Sabe-se que os principais fatores que influenciam nas atividades das bactérias metanotróficas são umidade, $\mathrm{pH}$, suprimento de oxigênio e temperatura.

$\mathrm{O} \mathrm{pH}$ e teor de sólidos voláteis ao longo da profundidade na camada metanotrófica no período estudado variou de 7,44 $\pm 0,85$ e $16,3 \% \pm 6,6 \%$ respectivamente. Jones e Nadell (1993) verificaram que solos com maior capacidade manutenção de umidade tais como os com alto teor de matéria orgânica, são mais favoráveis a reações de oxidação de $\mathrm{CH}_{4}$.

A concentração desde o contato solo/resíduo nas 15 medições, variou de $47 \%$ a $53 \%$ para o $\mathrm{CH}_{4}$, de $33 \%$ a $40 \%$ para o $\mathrm{CO}_{2}$, e de $0,3 \%$ a $5,4 \%$ para o $\mathrm{O}_{2}$. Na superfície as concentrações de $\mathrm{CH}_{4}$ variaram de $0 \%$ a $15 \%$, de $\mathrm{CO}_{2}$ variaram de $2,8 \%$ a $24,5 \%$ e de $\mathrm{O}_{2}$ variaram de $2,7 \%$ a $19,7 \%$.

A tabela 02 apresenta a eficiência na redução de concentração de $\mathrm{CH}_{4}$ desde o contato solo/resíduos até $10 \mathrm{~cm}$ da superfície, a profundidade onde se verificou a mudança brusca de concentração de $\mathrm{CH}_{4}$ e a respectiva temperatura e umidade nessa referida profundidade. 
Tabela 2 - Variação de concentração de metano, temperatura e umidade média em profundidade

\begin{tabular}{|c|c|c|c|c|c|}
\hline Data & $\begin{array}{c}\text { \% Eficiência } \\
\text { na } \\
\text { retenção/oxida } \\
\text { ção de } \mathrm{CH}_{4} \\
\end{array}$ & Prof.* (cm) & $\begin{array}{c}\text { Temperatura } \\
\left({ }^{\circ} \mathrm{C}\right)\end{array}$ & $\begin{array}{c}\text { Umidade } \\
\text { gravimétrica } \\
(\%)\end{array}$ & $\begin{array}{c}\% \mathrm{O}_{2} \\
\text { a } 10 \mathrm{~cm} \text { de } \\
\text { profundidade }\end{array}$ \\
\hline 03/set & $70,75 \%$ & 20 & 26,1 & $33,73 \%$ & 8,4 \\
\hline 09/set & $100,00 \%$ & 20 & 34,2 & $30,75 \%$ & 13,8 \\
\hline $16 /$ set & $54,60 \%$ & 10 & 31,1 & $33,51 \%$ & 16,6 \\
\hline $25 /$ set & $88,61 \%$ & 20 & 37,0 & $28,36 \%$ & 10,2 \\
\hline 06/out & $35,90 \%$ & 10 & 37,3 & $26,12 \%$ & 17,6 \\
\hline 13/out & $47,41 \%$ & 30 & 37,5 & $21,04 \%$ & 13,2 \\
\hline 28/out & $59,17 \%$ & 10 & 41,4 & $24,63 \%$ & 14,9 \\
\hline 05/nov & $71,05 \%$ & 20 & 43,9 & $22,91 \%$ & 17,6 \\
\hline 19/nov & $83,97 \%$ & 20 & 43,1 & $22,61 \%$ & 13,9 \\
\hline 10/dez & $65,10 \%$ & 20 & 39,6 & $23,28 \%$ & 14,8 \\
\hline 28/dez & $37,21 \%$ & 20 & 40,4 & $24,40 \%$ & 10,6 \\
\hline 12/jan & $56,13 \%$ & 10 & 36,5 & $22,61 \%$ & 7,1 \\
\hline 28/jan & $100,00 \%$ & 20 & 32,3 & $25,15 \%$ & 14,3 \\
\hline $12 / \mathrm{fev}$ & $34,89 \%$ & 10 & 35,9 & $23,88 \%$ & 2,7 \\
\hline 26/fev & $88,23 \%$ & 20 & 39,4 & $17,99 \%$ & 10,1 \\
\hline Média & $66,20 \%$ & & & & \\
\hline Desv. Pad. & $22,38 \%$ & & & & \\
\hline
\end{tabular}

$\mathrm{P}^{*}=$ profundidade de variação brusca de concentração de $\mathrm{CH}_{4}$

Os resultados indicam que a atividade de oxidação do $\mathrm{CH}_{4}$ pode estar ocorrendo na profundidade variando de 10 a $30 \mathrm{~cm}$ da superfície com predominância de $20 \mathrm{~cm}$. Essas variações na profundidade decorrem principalmente das variações de umidade, temperatura e aumento de teor de oxigênio no perfil do solo em virtude de variações climáticas (chuvas, pressão atmosférica e temperatura). Esses fatores também promovem o crescimento de vegetação que promove evapotranspiração controlando a umidade. Além disso, variações nas condições ambientais tais como na pressão atmosférica e precipitação também influenciam nas taxas de oxidação, pois o aumento da pressão promove maior entrada de $\mathrm{O}_{2}$ na camada de cobertura, e a precipitação promove mudanças na umidade do solo. Cabral, et. al. (2007), Huber-Humer, et. al. (2007) e Berger et. al. (2005) também constataram maior atividade de oxidação nos $30 \mathrm{~cm}$ superficiais da camada. Ainda de acordo com Huber e Lechner (2003), a microbiota metanotrófica pode se deslocar ao longo da profundidade na busca por melhores condições metabólicas.

A figura 6 mostra a eficiência em função da umidade gravimétrica e da temperatura, na profundidade de mudança brusca de concentração de $\mathrm{CH}_{4}$, em cada ensaio, em função da eficiência observada. 


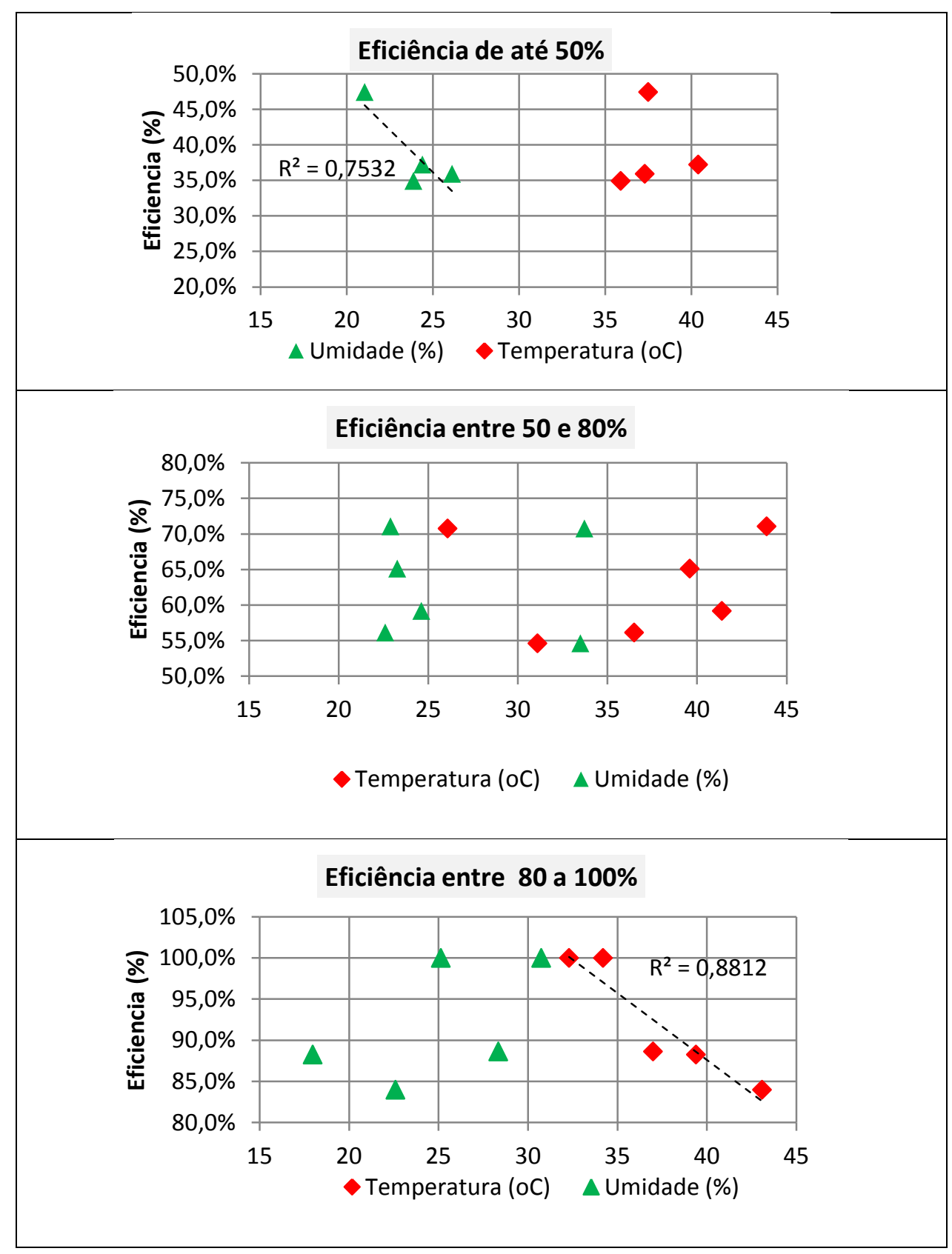

Figura 5 - Variação de umidade e temperatura com eficiência de oxidação

A literatura não é conclusiva sobre a faixa ótima de temperatura do solo para atividade de oxidação do $\mathrm{CH}_{4}$. Para esse caso as temperaturas mais altas diminuíram a eficiência de retenção/oxidação, sendo as maiores taxas de retenção/oxidação (100\%) observadas entre $32^{\circ} \mathrm{C}$ e $34^{\circ} \mathrm{C}$, enquanto que para as outras faixas de eficiência, a relação entre temperatura e eficiência não foi bem estabelecida. Gebert et. al. (2007) comprovaram que as taxas de oxidação crescem exponencialmente para temperaturas até $38^{\circ} \mathrm{C}$, embora tenha se verificado atividade oxidativa para toda faixa de temperatura analisada pelos autores $\left(3^{\circ} \mathrm{C}\right.$ até $45^{\circ} \mathrm{C}$ ). Nessa pesquisa as taxas de retenção oxidação variaram de $34,9 \%$ a $100 \%$ com faixas de temperatura entre $26,1^{\circ} \mathrm{C}$ a $43,9^{\circ} \mathrm{C}$.

$\mathrm{O}$ teor de umidade do solo na profundidade de variação brusca de concentração de $\mathrm{CH}_{4}$ variou de $18 \%$ a $33,7 \%$. A literatura menciona que deve existir uma faixa ótima para 
processos de oxidação em função da adaptação dos micro-organismos, pois esse processo é fortemente regulado pela umidade do material, principalmente através da difusão de $\mathrm{CH}_{4}$ no perfil do solo e pela atividade de bactérias metanotróficas. Hilger e Humer (2003) estudando aspectos geotécnicos relacionados com a oxidação de $\mathrm{CH}_{4}$ concluíram que a umidade ótima para captura desse biogás está em torno de $10 \%$ a $20 \%$. O fator crítico para que ocorra a oxidação seria, portanto, a porosidade que muda em função da umidade, pois o $\mathrm{CH}_{4}$ oriundo da biodegradação dos resíduos deve atingir os micro-organismos por difusão e se não houver porosidade suficiente não haverá favorecimento de reações de oxidação (AIT-BENICHOU et al., 2009).

Embora esse estudo tenha sido realizado em um período seco (setembro a fevereiro), a camada metanotrófica foi capaz de manter níveis de saturação acima de $50 \%$ o que indica uma capacidade de retenção de umidade, favorecida pelo teor de matéria orgânica e, consequentemente, presença constante de vegetação, como pode ser visto na figura 6 , ao contrário de uma camada convencional adjacente, na mesma área de pesquisa

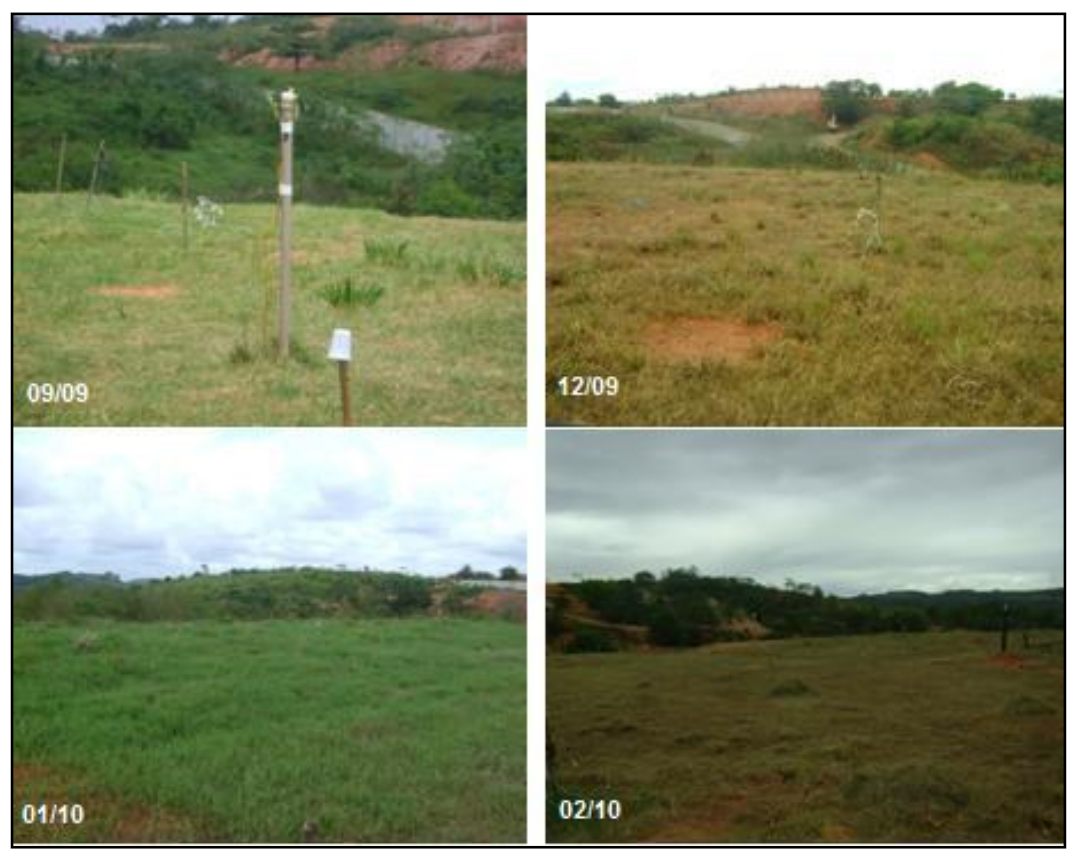

Figura 6 - Presença de vegetação na camada metanotrófica

De acordo com Huber-Humer et al. (2009) a utilização de materiais tais como composto, em camadas de cobertura, altera as propriedades específicas em relação ao um solo convencional, no que diz respeito a permeabilidade ao gás, aos parâmetros físicos, incluindo a capacidade de retenção de água e a textura e a atividade de oxidação do $\mathrm{CH}_{4}$, confirmando que o maior teor de matéria orgânica nesse tipo de camada favoreceu os altos índices de retenção/oxidação.

\section{CONCLUSÕES}


As camadas de cobertura do tipo metanotrófica ou oxidativas podem ser utilizadas como alternativas às camadas convencionais de solo compactado, para o gerenciamento de emissões de gases de efeito estufa em aterros sanitários.

Dos resultados obtidos ao longo dessa pesquisa se demonstra que as variações de emissões de $\mathrm{CH}_{4}$ nas camadas metanotróficas ( 0 a $151,95 \mathrm{~g} / \mathrm{m}^{2}$.dia) são inferiores (cerca de 6 vezes) em relação as camadas convencionais de acordo com resultados observados em Lopes et al. (2009) para a mesma célula experimental e de 3 vezes aos observados por Maciel (2003) e Mariano (2008) em camadas convencionais de aterros na mesma região.

Teores de umidade ao longo do perfil de profundidade variando de $18 \%$ a 33,7\% além de características químicas tais como $\mathrm{pH}$ acima da neutralidade e teor de matéria orgânica de $16,6 \%$ apresentados por essa camada metanotrófica, favorecem aos processos de oxidação e minimizam as emissões de $\mathrm{CH}_{4}$. A matéria orgânica facilita a agregação das partículas, conferindo estabilidade estrutural ao solo e formação de macro e microporos, responsáveis pela aeração e pela capacidade de retenção de água, o que favorece aos processos de oxidação, pois propicia ambiente para desenvolvimento de micro-organismos metanotróficos que oxidam $\mathrm{CH}_{4}$, assim como promove maior grau de saturação, que interfere no fluxo de gás, pela redução da permeabilidade.

Eficiência de retenção/oxidação de $\mathrm{CH}_{4}$ variou de $34,9 \%$ até $100 \%$ sendo determinados a partir de medições de perfil de concentração ao longo da espessura da camada. A profundidade onde se verificou diminuição brusca da concentração de $\mathrm{CH}_{4}$ com aumento na relação de $\mathrm{CO}_{2} / \mathrm{CH}_{4}$, variou de 10 a $30 \mathrm{~cm}$. A maior concentração de $\mathrm{O}_{2}$ observada também nesse intervalo de profundidade pode ter sido favorecida pelas propriedades geotécnicas da camada metanotrófica cuja granulometria mais arenosa facilita o fluxo de líquidos e gases com o meio externo, assim como pela presença da vegetação nessa camada, que facilita as entradas de $\mathrm{O}_{2}$ do ambiente através das raízes.

Dessa forma, pode-se concluir que as camadas metanotróficas se apresentam como alternativa promissora para redução da poluição atmosférica e minimização de emissões de $\mathrm{CH}_{4}$ em camadas de cobertura de aterros de resíduos sólidos, principalmente nos aterros onde a coleta e o aproveitamento econômico do biogás não são técnica e economicamente viáveis, tais como os de pequeno e médio porte e os aterros já encerrados com presença de resíduos mais antigos.

\section{AGRADECIMENTOS}

Os autores agradecem a CHESF pelo financiamento do projeto e a EMLURB pela participação na instalação da célula. A CAPES pela bolsa de doutorado do primeiro autor.

\section{REFERÊNCIAS BIBLIOGRÁFICAS}

1. AIT-BENICHOU, S.; JUGNIA, LOUIS-B.; GREER, C. W.; CABRAL, AL. R. (2009) Methanotrophs and methanotrophic activity in engineered biocovers. Waste Management v. 29, p 2509-2517 
2. BOGNER, J.E., SPOKAS, K.A., BURTON, E.A. (1997). Kinetics of Methane Oxidation in a Landfill Cover Soil: Temporal Variations, a Whole-Landfill Oxidation Experiment, and Modeling of Net CH4 Emissions, Environ. Sci. Technol., 31, 25042514.

3. BERGER, J.; FORNE`S, L.V.; OTT, C.; JAGER, J.; WAWRA, B.; ZANKE, U. (2005). Methane oxidation in a landfill cover with capillary barrier. Waste Management 25 (2005) 369-373.

4. BORJESSON, G.; SVENSSON B. (1997) Seasonal and diurnal methane emissions from a landfill and their regulation by methane oxidation. Waste Management and Research, 15(1), pp. 33-54.

5. CABRAL, A.; ARTEAGA, K.; RANNAUD, D.; AIT-BENICHOU, S.; POUET, M.F.; ALLAIRE, S.; JUGNIA, L.B.; GREER, C. (2007). Analysis of methane oxidation and dynamics of methanotrophies within a passive methane oxidation barrier, In: Proceedings Sardinia 2007, Eleventh International Waste Management and Landfill Symposium, Cagliari, Itália. CD

6. EMBRAPA (1999). Manual de análises químicas de solos, plantas e fertilizantes. Embrapa Solos, Embrapa Informática Agropecuária; organizador Fábio César da Silva. Brasília: Embrapa Comunicação para Transferência de Tecnologia. 370 p.

7. GEBERT, J.; GROENGROEFT, A.; MIEHLICH, G. (2007). Methane oxidation in a biofilter system - influence of water content, temperature and salt concentration. In Proceedings Sardinia 2007. Eleventh International Waste Management and Landfill Symposium S. Margherita di Pula. Cagliari. Italy. CD.

8. HUBER-HUMER, M.; LECHNER, P. A. (2003). Effect of methane oxidation on the water balance of the landfill cover and the vegetation layer. In: Proceedings Sardinia 2003. Ninth International Waste Management and Landfill Symposium, Cagliari, Itália, $\mathrm{CD}$

9. HUBER-HUMER, M.; PRANTIL, R.; LECHNER, P. (2007). Improvement of the upper waste layer to foster methane oxidation (2007) In: Proceedings Sardinia 2007, Eleventh International Waste Management and Landfill Symposium, Cagliari, Itália. CD

10. HUBER-HUMER, M.; GEBERT, J.; HILGER, H. A. (2008). Biotic systems to mitigate landfill methane emissions. Waste Management \& Research, Vol. 26, No. 1, pp 33-46.

11. HUBER-HUMER, M.; RODER, S.; LECHNER, S. (2009). Approaches to assess biocover performance on landfills. Waste Management 29, pp. 2092-2104.

12. IBGE (2008) Pesquisa Nacional de Saneamento Básico. Brasilia, DF. Disponível em < http://www.ibge.gov.br>. Acesso em 10 de setembro de 2010.

13. IPPC (2007). Climate Change 2007: Mitigation. Contribution of Working Group III to the Fourth Assessment Report of the Intergovernmental Panel on Climate Change. B. Metz, O. R. Davidson, P. R. Bosch, R. Dave, L. A. Meyer (eds), Cambridge University Press, Cambridge, United Kingdom and New York, NY, USA. Disponível em: <http://www.ipcc.ch/pdf/assessment-report/ar4/wg3/ar4-wg3-ts.pdf> Acesso em: 25 de maio de 2008.

14. KOERNER, R.M.; DANIEL, D. E. (1997). Final covers for solid waste landfills and abandoned dumps. $1^{\text {a }}$ ed. New York, ASCE PRESS. 256p. 
15. LOPES, R.L.; MACIEL, F. J.; JUCÁ, J.F.T.; NOGUEIRA, G. A. B. (2008). Avaliação da emissão de metano em aterro de resíduos sólidos experimental de pequeno porte na Muribeca/PE. $3^{\circ}$.Congreso Interamericano de Resíduos Sólidos de AIDIS. Buenos Aires.

16. MACIEL, F. J. (2003). Estudo da geração, percolação e emissão de gases no aterro de resíduos sólidos da Muribeca/PE. Dissertação de Mestrado. Universidade Federal de Pernambuco (UFPE). Recife-PE. 173 p.

17. MACIEL, F. J. (2009). Geração de biogás e energia em aterro experimental de resíduos sólidos urbanos. Tese de Doutorado. Universidade Federal de Pernambuco (UFPE). Recife-PE. 350p.

18. MACIEL, F.J.; LOPES, R.L.; JUCÁ, J.F.T. (2010). Evaluation of landfill gas emission in experimental cover layers in Brazil. In: Proceedings UNSAT 2010, Fifth International Conference Unsaturated Soil. Barcelona.Spain.

19. MARIANO, M. O. H. (2008). Avaliação da retenção de gases em camadas de cobertura de aterros de resíduos sólidos. Tese de Doutoramento. PPGEC-UFPE. Recife. 243p.

20. PERDIKEA, K.; MEHROTRA, A.K; PATRICK, HETTIARATCHI, J. P. A (2007). Study of thin biocovers (TBC) for oxidizing uncaptured methane emissions in bioreactor landfills. Waste Management. Article in Press.

21. ROSE, J.L.; GOUVEIA, P.P.F.; MAHLER, C. F. (2007). Study of methane oxidation on a covering layer. In: Proceedings Sardinia 2007, Eleventh International Waste Management and Landfill Symposium, S. Margherita di Pula, Cagliari, Italy.

22. SCHEUTZ, C.; BOGNER, J.; CHANTON, J.P; BLAKE, D.; MORCET, M.; KJELDSEN, P. (2003). Comparative oxidation and net emissions of CH4 and selected non-methane organic compounds in landfill cover soils. Environmental Science and Technology, 37, pp. 5143-5149.

23. URMANN, K.; NORINA, E. S.; SCHROTH, M. H. ZEYER, J. (2007). Methanotrophic activity in a diffusive methane/oxygen counter-gradient in an unsaturated porous medium. Journal of Contaminant Hydrology n. 94, p126-138.

24. VAN ELK, A. G. H. P. (2007). Redução de emissões na disposição final. In: Mecanismo de Desenvolvimento Limpo aplicado a Resíduos Sólidos Urbanos. Karin Segala (Coord.) Rio de Janeiro: IBAM

25. WILSHUSEN, J.H.; HETTIARATCHI, J.P.A.; DE VISSCHER, A.; SAINT-FORT, R., (2004). Methane oxidation and EPS formation in compost: effect of oxygen concentration. Environmental Pollution 23, 305-314. 